\title{
Effect of LASER on the Synthesis of Gold Nanoparticles with Reference to Geometries
}

\author{
Shweta Yadav, Soam Prakash \\ Department of Zoology, Faculty of Science, Environmental \& Advanced Parasitology \& Vector Control \& Biotechnology \\ Laboratories, Dayalbagh Educational Institute, Agra, India \\ Email: syshweta1@gmail.com
}

How to cite this paper: Yadav, S. and Prakash, S. (2018) Effect of LASER on the Synthesis of Gold Nanoparticles with Reference to Geometries. Advances in Nanoparticles, 7, 69-76.

https://doi.org/10.4236/anp.2018.74006

Received: July 31, 2018

Accepted: November 6, 2018

Published: November 9, 2018

Copyright (c) 2018 by authors and Scientific Research Publishing Inc. This work is licensed under the Creative Commons Attribution International License (CC BY 4.0).

http://creativecommons.org/licenses/by/4.0/

c) (i) Open Access

\begin{abstract}
Problem Statement: In the current study the effort was being made to investigate the effect of LASER light on the shape and geometries of Gold Nanoparticles. Light is an important parameter that plays significant role in the formation of nanoparticles; in this study LASER lights of selected wavelengths and colors have been used for the exposure of Gold Nanoparticles. The possibilities to manipulate the geometries of Gold Nanoparticles by altering the colors and wavelengths of LASER have been studied with reference to their efficacy against Culex quinquefasciatus. Approach: In the experimental setup four black boxes are used with no exposure to light sources. Three LASER with selected wavelengths and colors were fixed in the boxes at a specific angle, and then the nanoparticle solution was allowed to react. The micrographs of the Gold nanoparticles have been evaluated through the Transmission Electron Microscope (TEM). Results: The TEM images have shown formation of different shapes of nanoparticles due to exposure in different colors and wavelengths of LASER. Thus it explains that the wavelength and colour of the light plays a decisive role in the formation of the shapes and geometries of the nanoparticles.
\end{abstract}

\section{Keywords}

LASER, Gold Nanoparticles, Geometry, Characterisation

\section{Introduction}

Nanotechnology is defined as the "the designing, fabricating and characterization, production and application of structures, devices and systems by controlling shape and size at the nanoscale". Over the past three decades, nanotechnology has grown as a promising field to resolve the technological issues prevalent 
in various branches of science and technology. Further nanotechnology research has focused on understanding the correlation between the roles of different physical parameters the optical, electrical, and magnetic properties of nanomaterials with respect to their size, shape, and geometries. There have been several experimental reports on the optical properties of metal nanoparticles, including gold nanospheres, nanorods and nanoprisms, silver nanospheres, nanowires, and nanoprisms, copper nanospheres, aluminum nanospheres, bimetallic nanoparticles composite nanoparticles with a core-shell structure and nanoparticle chains and assemblies [1]. Manipulation of physico-chemical parameters viz. temperature, $\mathrm{pH}$ concentrations, and light has been a very interesting aspect of nanoparticle synthesis and heir significant effect on the shapes and sizes of the nanoparticles [2]. There are methods of synthesis of size and shape controlled nanoparticles by Laser ablation techniques [3], but in the current study the synthesized Gold nanoparticles are being exposed to different wavelengths and colors of Laser. This alteration plays a tremendous role in the formation of different geometries of Gold nanoparticles. Further their role in the efficacies against the selected mosquito species was also explored, which exhibited different efficacies for different geometries of nanoparticles. Optical properties of gold nanoparticles can thus be readily tuned by varying their size and shape by using their external physical parameters [1]. In the present investigation we are exploiting the selected wavelengths of Laser lights and the effect induced on the shapes of Gold nanoparticles was observed.

\section{Materials and Methods}

\subsection{Synthesis of Gold Nanoparticles}

Gold nanoparticles were synthesized with citrate-stabilized method [4]. For the preparation of citrate-stabilized seeds, aqueous solutions of $\mathrm{HAuCl}_{4}(0.01 \mathrm{M}$, $0.125 \mathrm{~mL})$ and citrate $(0.01 \mathrm{M}, 0.25 \mathrm{~mL})$ were added into water $(9.625 \mathrm{~mL})$, and then a freshly prepared, ice-cold aqueous $\mathrm{NaBH}_{4}$ solution $(0.01 \mathrm{M}, 0.15 \mathrm{~mL})$ was added under vigorous stirring. The prepared seed solution was kept at room temperature for at least $2 \mathrm{~h}$ before further use. The growth solution of Au nanoassemblies were prepared by the addition of $\mathrm{HAuCl} 4(0.01 \mathrm{M}, 1.8 \mathrm{~mL})$, and ascorbic acid $(0.1 \mathrm{M}, 0.3 \mathrm{~mL})$ aqueous CTAB solution $(0.1 \mathrm{M}, 42.75 \mathrm{~mL})$. The citrate-stabilized seed solution $(40 \mu \mathrm{L})$ was then added. The entire reaction solution was allowed to mix by gentle inversion for $10 \mathrm{~s}$ and then left for overnight.

\subsection{Synthesis of AuNPs in the Black Box Equipped with LASER Light}

Four black boxes of equal dimensions were kept and the LASER lights were suspended from the ceiling of the box and at the same orientation level in the each box. In every box the test tube containing the synthesized Gold nanoparticles were kept just below the suspended light so as the beam of LASER passes through the nanoparticle solution (Time of exposure of light to the solution will 
be same for all the test tubes. One box was taken completely dark which was used as the control).

\subsection{Exposure of Synthesized Nanoparticles under the LASER Beam}

The Gold nanoparticles that have been synthesised with the above mentioned procedure were kept under the LASER beam and a box tightly packed with the black paper sheets (with no slightest exposure of light. These boxes were kept undisturbed for 48 hours to allow the probable reaction to happen).

\section{Characterisation of the Synthesized Nanoparticles}

\section{UV-Vis Analysis}

For the characterization of the of the synthesized Gold nanoparticles was confirmed by sampling the reaction mixture at regular intervals and the absorption maxima was scanned by UV-Vis spectra, at the wavelength of $200-800 \mathrm{~nm}$ in UV-3600 Shimadzu spectrophotometer at $1 \mathrm{~nm}$ resolution Wavelength of peak absorbance, $\lambda \max$ was noted and the fwhm (full width at half-maximum) was calculated according to Herlekar et al. [5] AuNPs were then precipitated by the centrifuge at the bottom of the flask after the nanoparticles were reduced. This precipitate was then washed out with double distilled water and then further analyzed by transmission electron microscope. The samples of AuNPs synthesized were placed in a drop of reaction mixture on a copper grid and then allowed the mixture to get evaporated.

\section{Results and Discussions}

The UV-Vis spectra of the Gold nanoparticles show difference in the peak picks for different beams of LASER. In Figure 1 the peak pick can be seen at $340 \mathrm{~nm}$ of wavelength for 0.932 Absorbance Units, in Figure 2 the peak pick is at $329.5 \mathrm{~nm}$ for 0.924 Absorbance Units. For White light exposure there were multiple peaks as illustrated in the graph, highest peak was observed at $310 \mathrm{~nm}$ for $0.994 \mathrm{Ab}$ sorption Units and at $410 \mathrm{~nm}$ (Figure 3). In Figure 4 for the no exposure of light the peaks were noisy with Absorption maxima at $406 \mathrm{~nm}$. The difference in Absorption maxima depicts the change in the kinetics of the formation of Gold Nanoparticles which can be explored further.

Effect of wavelengths and colors of LASER on the geometries of Gold nanoparticles (TEM analysis)

In the present study 3 LASER of selected wavelengths and colors are used with the wavelengths $(280 \mathrm{~nm}, 320 \mathrm{~nm}, 560 \mathrm{~nm}$ ) respectively with the colors (green, red and white) and one dark box without light as control.

1) Green Light $(280 \mathrm{~nm})$ : The AuNPs formed after the exposure of green light were found to be spherical in shape with well defined crystalline lattices (Figure 5(a)).

2) Red Light $(320 \mathrm{~nm})$ : The AuNPs formed after the exposure of red light 


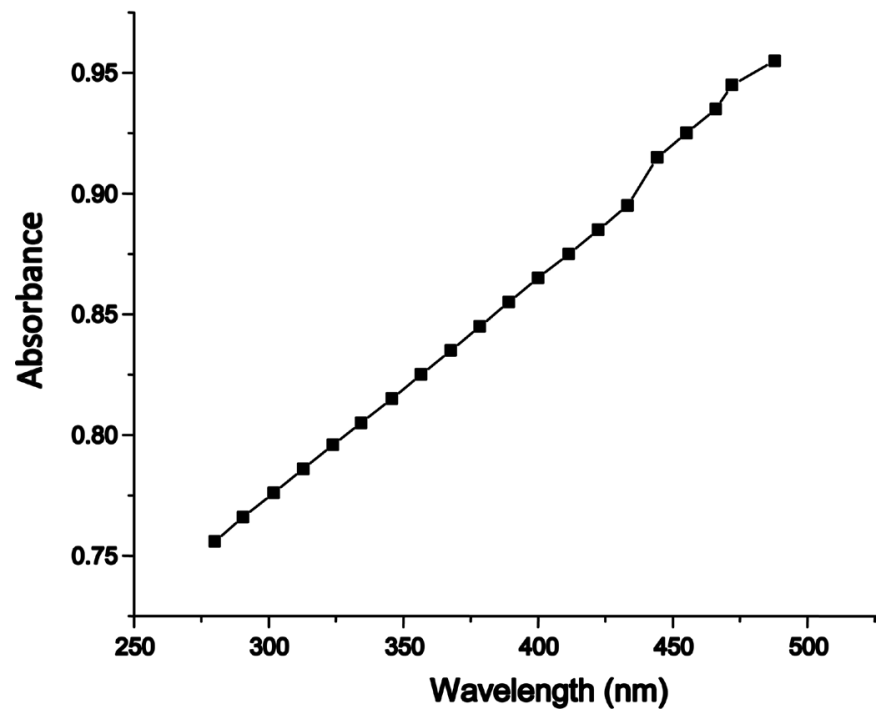

Figure 1. UV-Vis Spectra for the Gold Nanoparticles exposed in Green light.

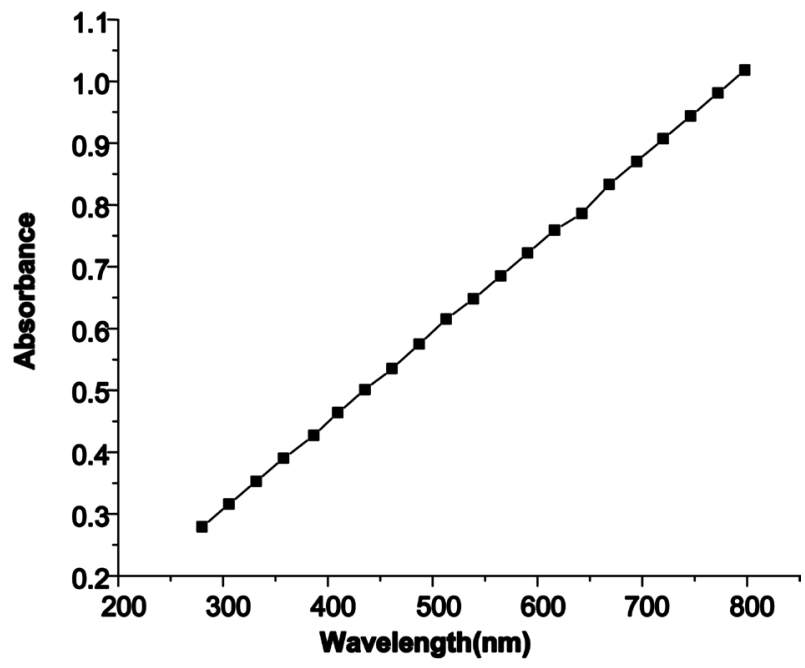

Figure 2. UV-Vis Spectra for the Gold Nanoparticles exposed under Red Light.

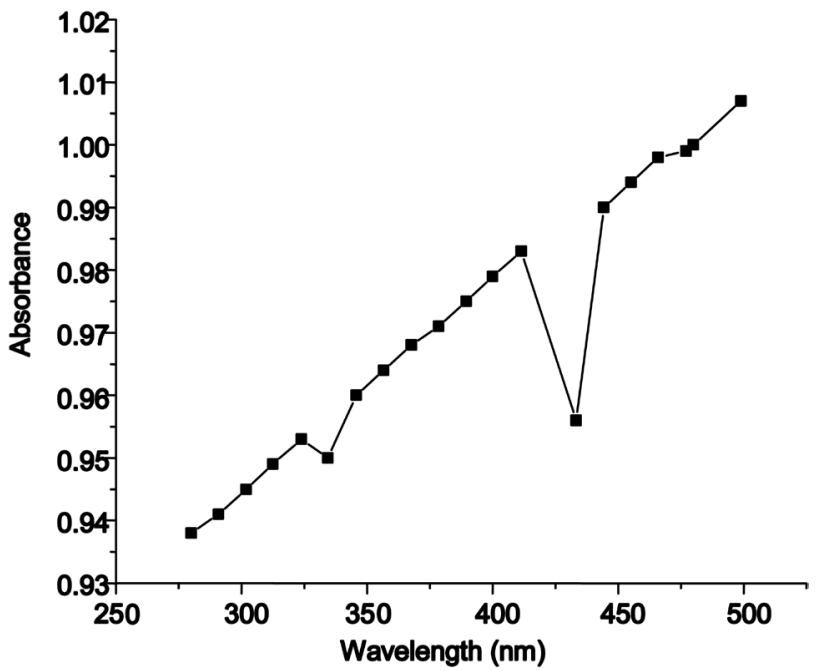

Figure 3. UV-Vis Spectra of Gold Nanoparticles (Exposure in White Light). 


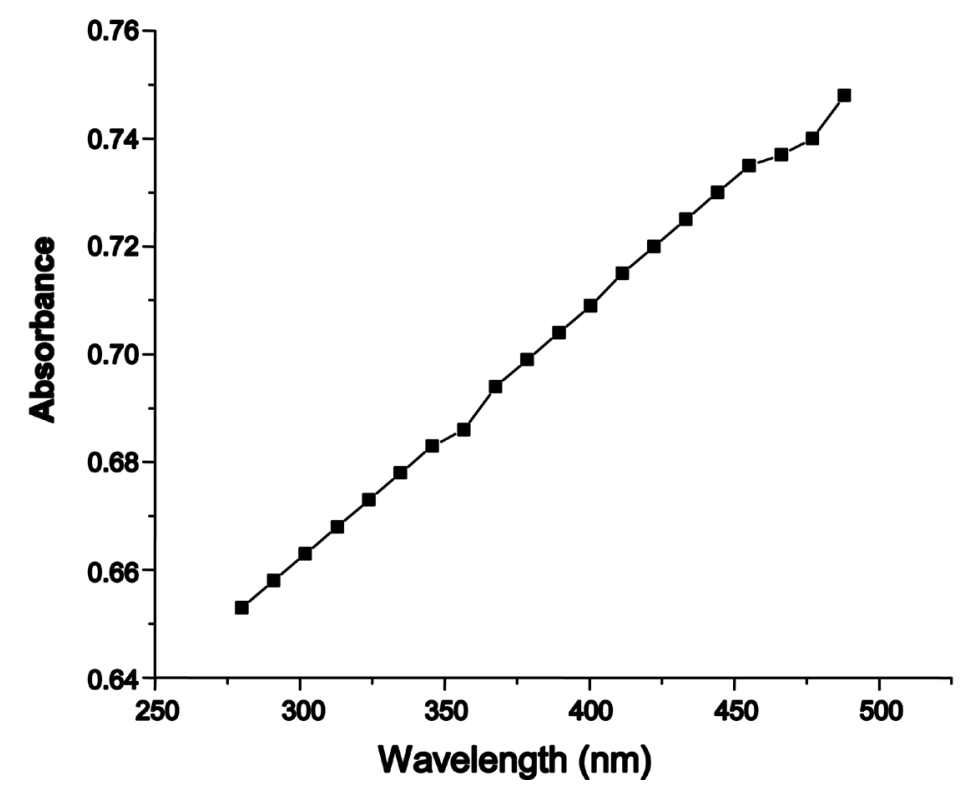

Figure 4. UV-Vis Spectra for Gold Nanoparticles (With no exposure to light).
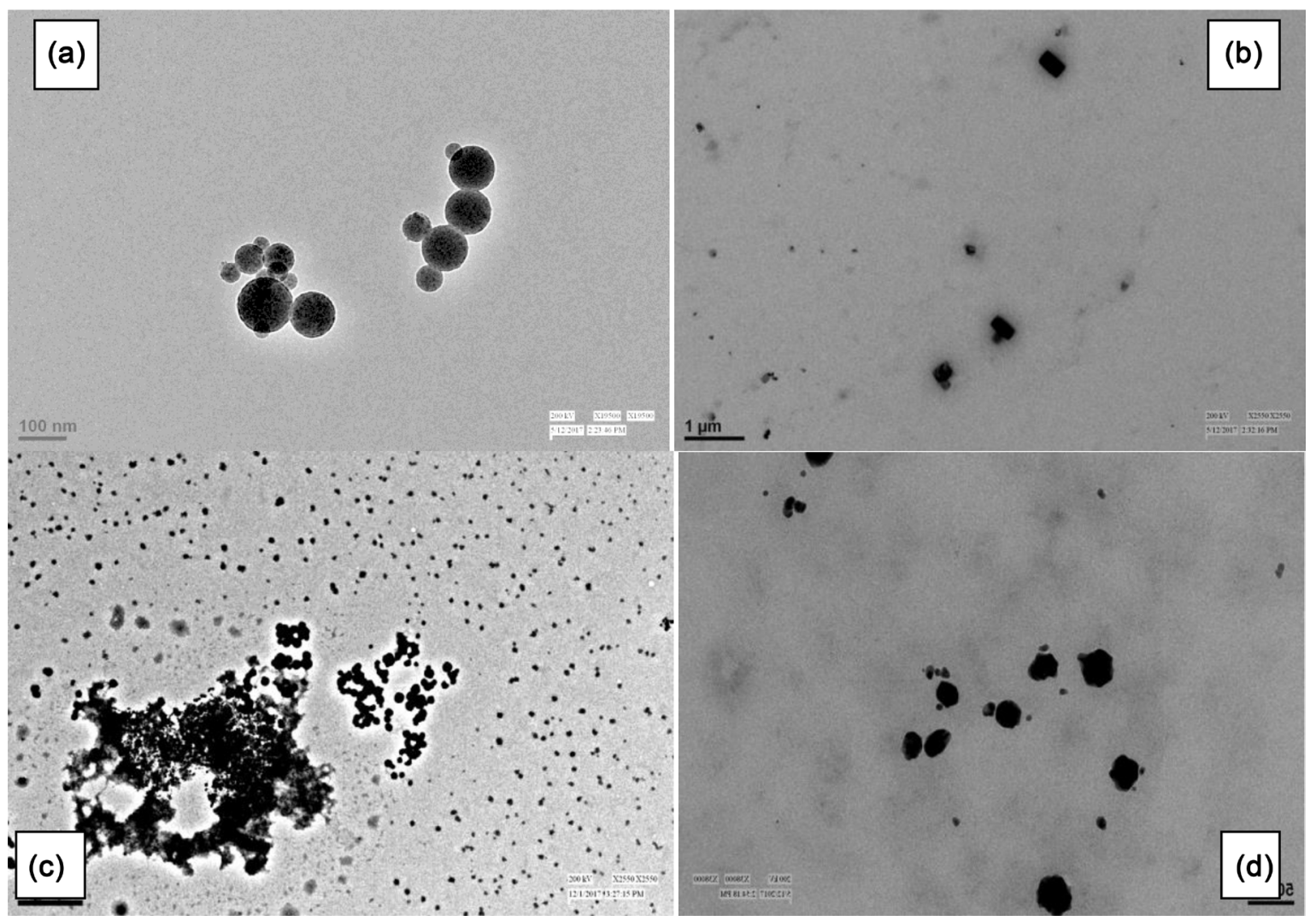

Figure 5. TEM Images depicting different shapes of Gold Nanoparticles when exposed under different beams of LASER (a) Spherical Gold Nanoparticles (Green light exposure) (b) Rectangular Gold Nanoparticles (Red light exposure) (c) Globular Gold Nanoparticles (White light exposure) (d) Irregular shaped Gold Nanoparticles (Dark box).

were found to be rectangular in shapes (Figure 5(b)).

3) White Light $(560 \mathrm{~nm})$ : The AuNPs formed after the exposure of white light were found to be globular in shape (Figure 5(c)). 
4) In the dark box: The nanoparticles which were kept under the dark after characterisation were observed to be of irregular shapes and geometries (Figure $5(d))$.

The efficacy of the synthesized AuNPs (synthesized under different LASER beams) was tested against the larvae of Culex quinquefasciatus. The larvae treated with the Nanoparticles synthesized under the Green LASER beams were found to be highly susceptible. The green light has shown $100 \%$ of mortality after 24 hours of exposure to the light. Efficacies under different lights after 24 hours of exposure were obtained (Table 1).

As the field of nanotechnology evolved, it is becoming clear that the particle shape is as important as size in determining the chemical and physical properties of a particular nanostructure [6]. This is especially found true for noble metal nanoparticles, where the plasmonic and catalytic properties can be finely tuned through control over their morphological properties play an important role in the formation of the shapes and geometries have studied the role of physical and chemical parameters like temperature, $\mathrm{pH}$ and have shown their role of spherical nanosilver and AuNPs for mosquito control [7]. Also they have used green methods for controlling mosquitoes by manipulating concentrations and time. Even they could be an effective adulticide which clearly show strong potential of these nanoparticles in controlling mosquito larvae adults of various species [8]. However their geometries can be exploited using nanoparticles have neither been experimented nor been exploited for mosquito control. With the advancement in the studies it has been seen that the physical parameters also play a very prominent role in the manipulation of the shapes and geometries of the nanoparticles [9]. Further the effect of controlled pulsed Laser can be investigated with their plasmonic resnonance properties over the formation of the nanoparticles. In a significant study a method has been proposed for the fabrication of a well defined size and shape distribution of Silver Nanoparticles, here they have directly employed the LASER irradiation method [10] whereas in the present paper we are using LASER pointers of specific wavelength. In a very significant study the nanoparticles production changes only with the laser parameters. and it was found out that the number of nanoparticles changes with the laser irradiation [11]. In recent days Laser ablation has emerged as the recent technique of synthesis of nanoparticles of selected shapes and geometries.

Table 1. Efficacy of synthesised Gold Nanoparticles under the exposure of different LASER beams.

\begin{tabular}{cccccc}
\hline Larval instar & Type of beam & $\begin{array}{c}\text { Wavelength } \\
(\mathrm{nm})\end{array}$ & $\begin{array}{c}\text { LC50 [ppm] } \\
(95 \% \mathrm{CI})\end{array}$ & $\begin{array}{c}\text { LC90 [ppm] } \\
(95 \% \mathrm{CI})\end{array}$ & $\begin{array}{c}\text { LC99 [ppm] } \\
(95 \% \mathrm{CI})\end{array}$ \\
\hline First & Green & 280 & $* *$ & $* *$ & $* *$ \\
Second & Red & 320 & $2(0.73-3.11)$ & $10(6.63-10.14)$ & $11(7.87-11.12)$ \\
Third & White & 560 & $1.5(1.36-2.64)$ & $9(7.77-10.23)$ & $10.5(9.17-11.83)$ \\
Fourth & Without Light & - & $3(2.86-4.13)$ & $11(8.72-12.23)$ & $12(10.64-12.16)$ \\
\hline
\end{tabular}


Physical parameters have played a very significant role in the change of the geometries of the nanoparticles. Light being an important parameter has also exhibited considerable change in the geometries. Effect of LASER on the shapes of Gold nanoparticles has been studied and it showed potential results. The femtosecond laser ablation of a gold target in aqueous solutions has been used to produce colloidal Au nanoparticles with controlled surface chemistry. A detailed chemical analysis showed that the nanoparticles formed were partially oxidized by the oxygen present in solution [12]. New method was being proposed for the fabrication of a well defined size and shape distribution of silver nanoparticles, this method employs direct laser irradiation, where as in our study we are directly exposing the solution to the laser beams and also with different colors of LASER changing geometries which is significant. A novel method for remote release of an encapsulated material from polyelectrolyte capsules is based on laser light illumination [13]. Applications of LASER induced beams in synthesis of nanoparticles have wide applications in biomedical field as well.

Presently in this work we have initiated the role of LASERS in initiating geometries which can be experimented further. In the present study we were able to experiment on the formation of AuNPs of specific geometrical shapes with the exposure of different LASER beams of selected wavelengths and colors. The light with specific wavelength $(280 \mathrm{~nm})$ and green color showed the formation of the spherical nanoparticles with size between 100 to $120 \mathrm{~nm}$. Light with wavelength $(320 \mathrm{~nm})$ red color exhibited the rectangular shaped nanoparticles of relatively smaller size. Light with wavelength $(560 \mathrm{~nm})$ showed slightly globular geometries with particle size ranging between $50 \mathrm{~nm}$ to $60 \mathrm{~nm}$. It was interesting to observe that the reaction mixture when kept inside black box (with no exposure to light) lead to the formation of irregularly shaped nanoparticles. The study provides desideration for further research and the application of newly derived geometries to have their utilization in disease control, drug testing and even as drug delivery.

\section{Conclusion}

We, therefore here would like to conclude that LASER beams with different wavelength and color could play a significant role in shaping geometries of AuNPs under synthesis and can be used for controlling Culex quiquefasciatus as shown in observations.

\section{Acknowledgements}

The authors profusely thank Prof P.S. Satsangi Sahab, Chairman of the Advisory Committee on Education at the Dayalbagh Educational Institute, Dayalbagh, Agra, India for support. Prof. Soami Piara, Head USIC for availing the FTIR facility. We express our sincere thanks and gratitude to SAIF, AIIMS for extending the Transmission Electron Microscopy (TEM) facility. 


\section{Conflicts of Interest}

The authors declare no conflicts of interest regarding the publication of this paper.

\section{References}

[1] Jain, P.K., Lee, K.S., El-Sayed, I.H. and El-Sayed, M.A. (2006) Calculated Absorption and Scattering Properties of Gold Nanoparticles of Different Size, Shape, and Composition: Applications in Biological imaging and Biomedicine. The Journal of Physical Chemistry B, 110, 7238-7248. https://doi.org/10.1021/jp057170o

[2] Kumari, M., Mishra, A., Pandey, S., Singh, S.P., Chaudhry, V., Mudiam, M.K.R. and Nautiyal, C.S. (2016) Physico-Chemical Condition Optimization during Biosynthesis Lead to Development of Improved and Catalytically Efficient Gold Nano Particles. Scientific Reports, 6, Article No. 27575. https://doi.org/10.1038/srep27575

[3] Mafuné, F., Kohno, J.Y., Takeda, Y. and Kondow, T. (2002) Full Physical Preparation of Size-Selected Gold Nanoparticles in Solution: Laser Ablation and Laser-Induced Size Control. The Journal of Physical Chemistry B, 106, 7575-7577. https://doi.org/10.1021/jp020577y

[4] Chen, H., Kou, X., Yang, Z., Ni, W. and Wang, J. (2008) Shape- and Size-Dependent Refractive Index Sensitivity of Gold Nanoparticles. Langmuir, 24, 5233-5237. https://doi.org/10.1021/la800305j

[5] Herlekar, M., Barve, S. and Kumar, R. (2014) Plant-Mediated Green Synthesis of Iron Nanoparticles. Journal of Nanoparticles, 2014, Article ID: 140614. https://doi.org/10.1155/2014/140614

[6] Zhang, J., Langille, M.R., Personick, M.L., Zhang, K., Li, S. and Mirkin, C.A. (2010) Concave Cubic Gold Nanocrystals with High-Index Facets. Journal of the American Chemical Society, 132, 14012-14014. https://doi.org/10.1021/ja106394k

[7] Prakash, N.S. and Soni, N. (2011) Factors Affecting the Geometry of Silver Nanoparticles Synthesis in Chrysosporium tropicum and Fusarium oxysporum. American Journal of Nanotechnology, 2, 112-121

[8] Soni, N. and Prakash, S. (2012) Fungal-Mediated Nano Silver: An Effective Adulticide against Mosquito. Parasitology Research, 111, 2091-2098. https://doi.org/10.1007/s00436-012-3056-x

[9] Soni, N. and Prakash, S. (2014) Microbial Synthesis of Nanosilver and Nanogold for Mosquito Control. Annals of Microbiology, 64, 1099-1111. https://doi.org/10.1007/s13213-013-0749-Z

[10] Abid, J.P., Wark, A.W., Brevet, P.F. and Girault, H.H. (2002) Preparation of Silver Nanoparticles in Solution from a Silver Salt by Laser Irradiation. Chemical Communications, No. 7, 792-793. https://doi.org/10.1039/b200272h

[11] Eliezer, S., Eliaz, N., Grossman, E., Fisher, D., Gouzman, I., Henis, Z. and Lereah, Y. (2004) Synthesis of Nanoparticles with Femtosecond Laser Pulses. Physical Review $B, 69$, Article ID: 14411. https://doi.org/10.1103/PhysRevB.69.144119

[12] Sylvestre, J.P., Poulin, S., Kabashin, A.V., Sacher, E., Meunier, M. and Luong, J.H. (2004) Surface Chemistry of Gold Nanoparticles Produced by Laser Ablation in Aqueous Media. The Journal of Physical Chemistry B, 108, 16864-16869. https://doi.org/10.1021/jp047134+

[13] Sukhorukov, G.B., Rogach, A.L., Garstka, M., Springer, S., Parak, W.J., Muñoz-Javier A. and Palankar, R. (2007) Multifunctionalized Polymer Microcapsules: Novel Tools for Biological and Pharmacological Applications. Small, 3, 944-955. https://doi.org/10.1002/smll.200600622 